\title{
HIPPARCOS AND THEORY OF STELLAR INTERIORS
}

\author{
What is being learned?
}

\author{
A. BAGLIN \\ DESPA, Observatoire de Paris, URA CNRS 264, \\ 5 place J. Janssen, Meudon CEDEX, France.
}

\section{Introduction}

The theory of stellar interiors is generally considered as well settled, and many branches of astrophysics rely on its results. However, the true situation is not so bright. Despite great successes at its beginning, in the sixties, it has to deal with many physical processes, generally poorly understood, which have large influences on the results and the derived quantities, like for instance ages.

After a rapid description of these difficulties, I will recall the major questions of the confrontation between theory and observations, and how HIPPARCOS can and will help. As an illustration, a few preliminary results will be presented, and future prospects foreseen. But, this short review is by no means exhaustive; there are many more results dealing directly or indirectly with stellar structure theory which have already been presented at the Venice Symposium (Perryman et al., 1997a), and many more, I am not aware of, will certainly appear soon.

\section{The Present Status of Internal Structure Theory}

This question is developed in many text books, and a recent critical review can be found in (Lebreton and Baglin, 1995). Standard stellar modelling rely, up to now, on hydrostatic models neglecting most of the hydrodynamical processes or replacing them by a very crude phenomenology. The microscopic description of the stellar material is generally safer, and has been seriously improved in the recent years. Several observational facts find no explanation in this framework showing that something is missing in the description.

The present major uncertainties concern hydrodynamical processes, like the treatment of the energy transport in convective regions, the extension of the convectively mixed regions, either convective cores or outer convective envelopes, and the transport of chemicals and momentum in radiative zones. Models are still generally ignoring the non axisymetric processes, as for instance rotation, and the hydrodynamical instabilities that they produce; see i.e. Zahn (1995), Schatzman (1995), Roxburgh (1997).

One of the major problem in testing the physical processes by confronting observational data with predicted ones, is that, for an individual object, the number of observables is generally smaller than the number of unknown quantities. Then, this comparison accepts a large variety of solutions, and additional hypothesis are needed. Dealing with 'homogeneous' ensemble of objects, like clusters or binaries, helps to reduce this indetermination. But, increasing the number of observables, for instance measuring radii and eigenmode frequencies, as expected, is the only way to solve the question.

In this comparison, the determination of the total luminosity is of great importance. Up to now, except for a few very nearby objects, the accuracy of direct determinations was too small to be useful, and one had to rely on photometric calibrations. HIPPARCOS, as expected, contributes very significantly in reducing these error bars in the Hertzprung-Russell diagram (Baglin, 1988).

\section{The solar-like non evolved objects and the convection parameter.}

These stars have convective envelopes and radiative interiors; they are slow rotators, and their evolutionary time scale is long, so that most of them are still on the main sequence. The treatment 
of the convective transport remains very rough though progress is underway, and up to now the available phenomenological treatments contain an almost free parameter. But the precise slope of the intermediate zero age main sequence $\left(0.6 \leq M_{\odot} \leq 1\right)$ can help.

Assuming homogeneity of the chemical composition and of the date of formation, ther position in the HR diagram depends essentially on the efficiency of the convective transport in the external outerlayers, which governs the temperature gradient, i.e. the radius and then the effective temperature. In the classical phenomenological formalism, the mixing length parameter $\alpha$ determines the slope of the main sequence (Fernandes, 1997). This slope is now well defined observationally, and common to many different objects, the Hyades and several nearby open clusters (Mermilliod et al., 1997), the field stars and subdwarfs, for which effective temperature and metal content are well constrained by detailed spectrocopic analysis and luminosities are derived from HIPPARCOS. Jt is compatible with a constant value of $\alpha$, in the region between 1 and $0.6 M_{\odot}, \alpha=\alpha_{\odot}$, where $\alpha_{\odot}$ is the value derived from the solar calibration, using the same physical description of the interior.

This result is valid for a large interval of metal content $Z$, but has not been checked for giants. Equivalent results are obtained with the more refined FST description (Canuto et al., 1996).

On can also gain a lot by calibrating nearby visual binaries, for which individual masses, luminosities and effective temperatures, as well as their common metal content $Z$ are measured. As, for these objects the number of unknown quantities is equal to the number of free parameters, a calibration similar to the one conducted for the Sun is possible (Noels et al., 1991); ages, helium contents, and $\alpha$ can be determined. Once more, with the same standard physics (Fernandes et al., 1997), the same value $\alpha_{\odot}$ is obtained, confirming the previous statement.

HIPPARCOS plays a crucial role here in increasing both the accuracy of the masses of already well known systems, and the number of double stars with accurate masses (Martin and Mignard, 1997), (Soderhjhelm et al., 1997).

These results, showing that the phenomenological scaling of the treatment of the superadiabatic layers of outerconvective zones is now settled, allow to go a step further. It becomes now possible to determine the Helium content $Y$ for groups of non evolved objects, as i.e. the Hyades cluster (Perryman et al., 1997b), and several nearby open clusters (Robichon et al., 1997). Ages of objects where $Y$ is known, i.e. old subdwarfs, can also be estimated as a function of $Y$.

\section{Position of the main sequence and metallicity}

The drastic reduction of the uncertainty on luminosity allows now to built representative sets of 'well known' object, sorted rigourously to avoid pecularities, and for which detailed spectroscopic analysis reaches its best quality. With these criteria, sets are quite small; i.e., starting wih a sample of more than 160 candidates of main sequence stars of spectral type between $F$ and $K$, with $-1 \leq\left[\frac{F_{e}}{H}\right] \leq 0.2$, only 38 satisfy the quality criteria necessary to make significant tests in the HR diagram, in particular $\sigma(\pi) / \pi \leq 0.05$ (Lebreton et al., 1997).

For 'solar' metallicity, the width of the main sequence is quite small, in favor of a large value of the galactic enrichment ratio $\frac{\Delta Y}{\Delta Z}$. But a difficulty appears for stars with low metallicity $-1 \leq\left[\frac{F_{e}}{H}\right] \leq-0.45$. Standard models with this metallicity could occupy such a position only if their helium content $Y$ was smaller than the primordial value. To solve this problem, a shift towards lower effective temperature of $\sim 250$ ? $\mathrm{K}$, or towards higher luminosities of $0.2 \mathrm{mag}$ is needed. And it seems to be definitely too large to be attributed to observational errors.

Going to the closest subdwarfs, $\left[\frac{F_{c}}{H}\right] \leq-1.0$, the sample is even more restricted, but the main sequence is well defined (Pont et al., 1997). The same kind of difficulty arises, with a slightly smaller, but still significant amplitude: the shift in temperature is only of $\sim 150$ ? $K$, and in luminosity of 0.15, (Cayrel et al., 1997).

If this effect is real, and not due to some uncertainties in the observed parameters or in the physical description, it looks as if the surface abundances ( $Z$ and/or $Y$ ) were not representative of the interior abundances. We already know from the Sun, that elements sedimentation seems to occur in radiative zones. But we also know that a complex interplay between sedimentation and mixing of the material is at work, and that its influence on stellar evoution can be important.

The present observed surface mixture would not represent the initial material from which the star was formed; it would be the result of a complex time dependent process, which has now to be understood. 
If these results are confirmed, HIPPARCOS will contribute to built this new refined description of stellar interiors.

\section{Dwarf $A / F$ stars of the instability strip}

For these stars, the main questions are the extension of convective core and the influence of rotation. Both have consequences on the age determinations. Improved luminosities from HIPPARCOS, show that, in the average they are brighter than predicted by the previous calibrations, though a large scatter exists (Liu et al., 1997), (Høg and Petersen, 1997). It has been also shown that variables, non variables and peculiar stars occupy the same domain of the HR diagram (North et al., 1997).

Unfortunately, the size of the convective core cannot be measured with the HR diagram alone. As most of these objects are pulsating, seismology will be the appropriate tool. But to be applied succesfully, it needs an excellent knowledge of the basic parameters of the object, which is reached presently only for the closest ones (Lebreton et al., 1995), and also a correct treatment of rotation. For instance, the new position of the binary system $\theta^{2}$ Tau in the Hyades, shifts significantly the predicted eigenfrequencies $(\sim 100 \mu \mathrm{Hz})$, and consequently the interpretation of the observed spectrum.

\section{Prospects}

This very preliminary and uncomplete sketch shows already that the HIPPARCOS mission, combined with progresses in other domains, contribute to the renewal of stellar structure and evolution theory. Convergence of better physics and observations raises new problems to fundamental physics and cosmology. In the domain of astrophysics, it is demanding more to stellar atmosphere theory and data acquisition of spectroscopic parameters which are now limiting the accuracy of the $\mathrm{Ob}$ servation/Theory confrontation.

But, one has to remind that the domain in which HIPPARCOS is largely improving the accuracy on luminosities remains very limited:

$$
\frac{\Delta L}{L} \sim 2 \frac{\sigma(\pi)}{\pi} \leq \delta(B . C .), \text { if } \pi \leq 10 \text { parsecs }
$$

where $\delta(B . C$.$) is the uncertainty on the bolometric correction.$

This is why one forsees already a need for even higher accuracy parallaxes to obtain higher precision for a few closeby objects, as well as the extension of the present accuracy to a larger sample of stars, and also to rare types of objects.

\section{References}

Baglin, A.(1987) in Scientific aspects of the Input Catalogue Preparation II, J. Torra and C. Turon eds, p. 103. Canuto,V.M., Goldman,I., Mazitelli, I.(1996) Astrophys. J., 473, 550.

Cayrel, R., Lebreton, Y., Perrin, M.N., Turon, C. (1997), Hipparcos symposium Venice, ESA publ. SP-402, p. 219. Fernandes, J. (1997) Ph. D. Thesis, Paris VII University

Fernandes, J., Lebreton, Y., Baglin, A. (1997), Astron. Astrophys, in press.

Høg, E., Petersen, J.O.(1997), Astron. Astrophys., in press.

Lebreton, Y., Michel, E., Goupil, M.J., Baglin, A., Fernandes, J. (1994), IAU Symposium 166, p.135.

Lebreton, Y., Baglin, A.(1995) in Stellar evolution, what should be done? 32th Liege Inst. Astroph. Coll, p.1.

Lebreton, Y., Perrin, M.N., Fernandes, J. et al. (1997) Hipparcos symposium Venice, ESA publ. SP-402, p. 379.

Liu, Y.Y, Baglin, A., Auvergne, M. et al. (1997) Hipparcos symposium Venice, ESA publ. SP-402, p. 363.

Martin C, Mignard F. (1997), Astron. Astrophys, in press

Mermilliod, J.C., Turon, C., Robichon, N. et al. (1997), Hipparcos symposium Venice, ESA publ. SP-402, p. 643.

Noels, A., Grevesse, N., Magain, P., Neuforge, C., Baglin A., Lebreton, Y. (1991), Astron, Astrophys, $247,91$.

North, P., Jaschek, C., Egret, D. (1997) Hipparcos symposium Venice, ESA publ. SP-402, p. 367.

Perryman M.A.C. et al. (1997a), Hipparcos symposium Venice, ESA publ. SP-402,

Perryman, M.A.C., Brown, A.G.A., Lebreton, Y. et al. (1997b), Astron. Astrophys., in press

Pont, F., Charbonnel, C., Lebreton, Y. et al. (1997), Hipparcos symposium Venice, ESA publ. SP-402, p. 699.

Robichon, N., Arenou, F., Turon, C., et al. (1997), Highlights in Astronomy, 11, in press.

Roxburgh, I.W.,(1997) IAU symposium 185, in press.

Schatzman, E..(1995) in Stellar evolution, what should be done? 32th Liege Inst. Astroph. Coll, p.121.

Soderhjelm, S., Lindengren, L., Perryman, M.A.C. (1997), Hipparcos symposium Venice, ESA publ. SP-402,p.251.

Zahn, J.P.(1995) in Stellar evolution, what should be done? 32th Liege Inst. Astroph. Coll, p.111. 